\title{
Marginal zone B-cell lymphoma of the pulmonary mucosa-associated lymphoid tissue: A case report
}

\author{
HONG LI, TING WANG, XIAOHONG WEI and XIAOMIN DANG \\ Department of Respiratory and Critical Care Medicine, The First Affiliated Hospital of Xi'an JiaoTong University, \\ Xi'an, Shaanxi 710061, P.R. China
}

Received February 24, 2014; Accepted November 21, 2014

DOI: $10.3892 / \mathrm{ol} .2015 .3375$

\begin{abstract}
Marginal zone B-cell lymphoma of the pulmonary mucosa-associated lymphoid tissue (pulmonary MALT-MZL) is a common type of primary pulmonary lymphoma, but is rare as a pulmonary malignant tumor. In the present study, a 49-year-old male patient was admitted to The First Affiliated Hospital of Xi'an JiaTong University (Xi'an, China) with a pulmonary lesion in the right upper lung. The patient was diagnosed with pulmonary MALT-MZL subsequent to undergoing chest computed tomography (CT), a routine blood test, pathological and histological examinations, a transbronchial lung biopsy and bronchoscopy. A chest CT scan revealed right middle lobe consolidation and inflammatory signs, accompanied by mediastinal lymphadenopathy in the anterior basal segment of the upper lobe and CT angiogram signs. Bronchial stenosis and swollen mucosa were observed by bronchoscopy. The tissue section of the transbronchial lung biopsy specimens revealed diffusely infiltrated monocytoid B-cell lymphocytes and a lymphoepithelial lesion. The tissue was found to be positive for cluster of differentiation (CD)20, B-cell lymphoma 2 and CD79a expression, but negative for CD3, CD5, cyclin D1 and $\kappa$-light chain expression. CD21 and CD23, located in the residual follicular dendritic cells, were detected by immunohistochemical staining. The clinical manifestations of pulmonary MALT-MZL are non-specific and misdiagnosis frequently occurs in clinical practice. Therefore, an appropriate invasive biopsy procedure is necessary for early and accurate diagnosis of pulmonary MALT-MZL. Clinical presentation that includes periodic fever and distended bronchi in pulmonary consolidation may indicate a diagnosis of MALT-MZL. Pulmonary MALT-MZL belongs to the category of indolent lymphoma and accurate clinical diagnosis is challenging. The
\end{abstract}

Correspondence to: Dr Xiaomin Dang, Department of Respiratory and Critical Care Medicine, The First Affiliated Hospital of Xi'an JiaoTong University, 277 Yanta West Road, Xi'an, Shaanxi 710061, P.R. China

E-mail: 1hdoccn@163.com

Key words: B-cell lymphoma, pulmonary malignant tumors, lymphoid tissue, diagnosis results in the present study may provide additional evidence for the accurate diagnosis of this rare entity.

\section{Introduction}

Extranodal marginal zone B-cell lymphoma (MZL) of mucosa-associated lymphoid tissue (MALT), and splenic and nodal MZL are the three subtypes of MZL recognized by the World Health Organization (WHO) classification (1). Overall, MZLs share morphological and immunophenotypical features that are similar to those of the hypothesized common cell of origin, the marginal zone B-cell in secondary B-follicles.

Extranodal MZL, also termed MALT lymphoma, is a distinct subgroup of non-Hodgkin's lymphoma (NHL) that accounts for $\sim 5 \%$ of all NHLs $(1,2)$. Despite the stomach being the most frequently involved organ in extranodal MZL, the lung is one of the most common non-gastrointestinal sites of occurrence $(3,4)$. Extranodal MZL accounts for $<0.5 \%$ of all primary lung neoplasms, but more than two-thirds of primary pulmonary NHLs are extranodal MZLs (5-7). Pulmonary MALT-MZL exhibits no clear symptoms and thus, the disease is usually identified during routine chest X-ray examinations (8). Pulmonary MALT-MZL is diagnosed by imaging examination and biopsy $(9,10)$ and the main treatment modalities for MALT-MZL include surgery, radiotherapy and chemotherapy $(11,12)$. Pulmonary MALTMZL exhibits a good prognosis, with a five-year survival rate of $93.6 \%(8,13)$.

Due to the non-specific clinical manifestations of pulmonary MALT-MZL, misdiagnosis is frequently observed in clinical practice. The present study describes the presentation and diagnosis of one patient with pulmonary MALT-MZL, and aims to provide evidence for the accurate diagnosis of pulmonary MALT-MZL. Written informed consent was obtained from the patient.

\section{Case report}

A 49-year-old male was referred to The First Affiliated Hospital of Xi'an JiaoTong University (Xi'an, Shaanxi, China) with a pulmonary lesion in the right upper lung observed on a chest radiograph that was performed during a routine health checkup at a local hospital. The patient had smoked for 


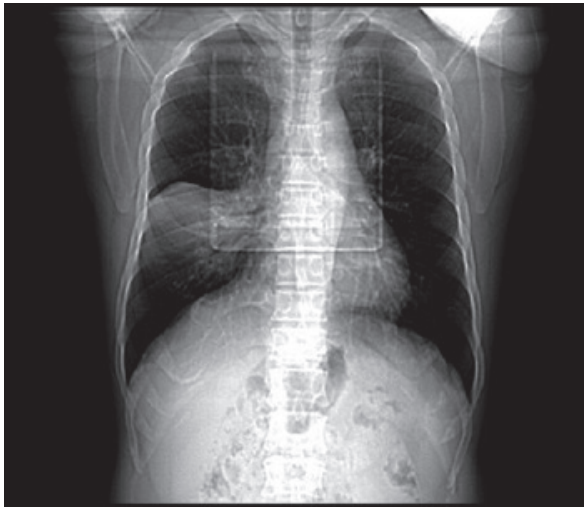

Figure 1. Chest radiograph revealing a lesion in the right upper lung.

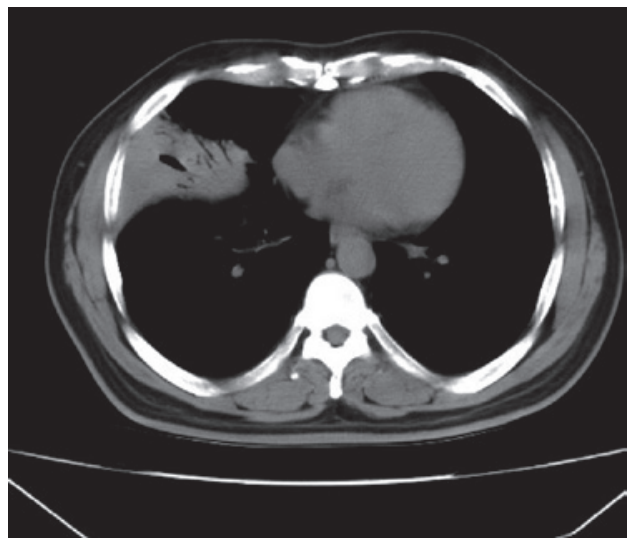

Figure 2. Chest computed tomography revealing a lesion in the anterior segment of the upper lobe of the right lung.
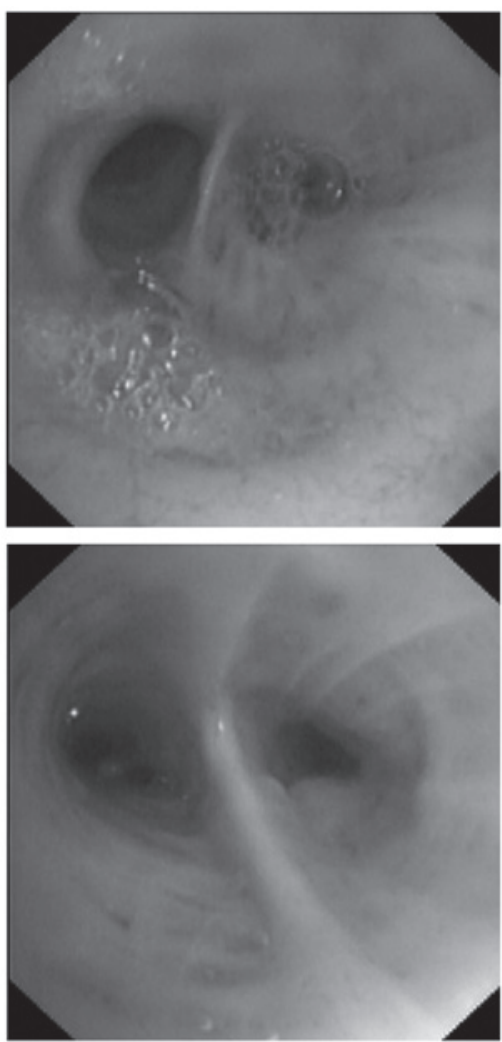

Figure 3. Bronchoscopy revealing mucosal edema and mild stenosis in the right main bronchus.

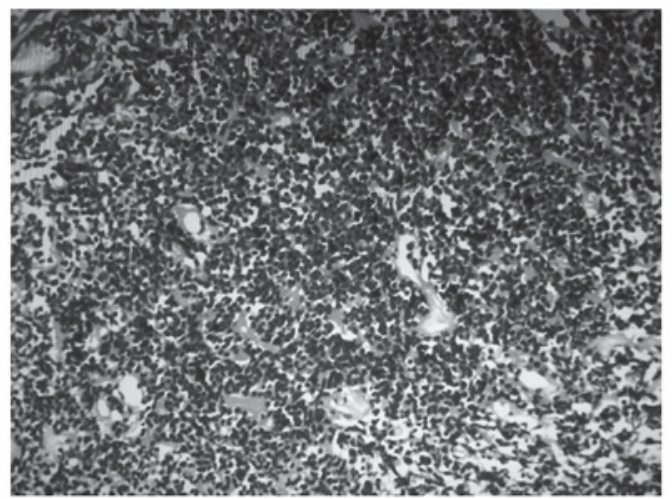

Figure 4. Immunohistochemical staining revealing positivity for CD20, B-cell lymphoma 2 and CD79a (magnification, x20).

$>20$ years and reported no history of respiratory illness, such as tuberculosis. The vital signs of the patient were normal, and a physical examination revealed no abnormalities.

The laboratory data were within the normal ranges, with the exception of a tumor marker, carcinoembryonic antigen (CEA), which possessed a level of $5.24 \mathrm{ng} / \mathrm{ml}$ (normal range, 0-3.4 ng/ml). Pulmonary function testing revealed no ventilatory defects. A chest radiograph revealed a nodule in the right upper lung (Fig. 1). A chest computed tomography (CT) scan revealed signs of inflammation accompanied by mediastinal lymphadenopathy in the anterior segment of the right upper lobe (Fig. 2). Additionally, flexible bronchoscopy was performed to identify the endobronchial lesion. At bronchoscopy, mucosal edema and slight stenosis were observed in the right main bronchus (Fig. 3). Immunohistochemical staining revealed diffuse strong positive staining for cluster of differentiation (CD)20, B-cell lymphoma-2 and CD79a, and the samples were negative for CD3, CD5, cyclin D1 and $\kappa$-light chain expression (Fig. 4). Considering the clinical outcomes, the pulmonary lesion was diagnosed as extranodal MALT-MZL. No evident absorption of the lesion was identified on CT scan following combined treatment with mezlocillin and sulbactam (3.75 g every $8 \mathrm{~h}$ ) for 1 week. Bronchoscopic examination and pathological analysis revealed no evidence of bleeding or lumps, which confirmed the diagnosis of extranodal MALT-MZL. Subsequently, the patient was transferred to the Hematology Department, but refused further treatment. No follow-up examinations were performed.

\section{Discussion}

In 1983, Isaacson and Wright (14) reported two cases of MALT lymphoma arising from the gastrointestinal tract for the first time. Subsequently, a variety of studies identified additional MALT lymphoma cases worldwide $(3,14,15)$. MALT lymphoma has been classified in the WHO and revised European-American classifications of lymphoid neoplasms as extranodal MALT-MZL $(1,4,7)$. This extranodal lymphoma with low-grade features infiltrates the marginal zone of reactive B-cell follicles and arises most commonly from the MALT of the stomach, but also from the lungs, salivary glands and thyroid gland (4). 
Pulmonary extranodal MZL is a rare entity that accounts for $<0.5 \%$ of primary pulmonary malignancies and $<1 \%$ of all lymphomas (5-7). The incidence of lung adenocarcinoma, which usually arises from peripheral small bronchi or alveolar epithelial cells, is increasing worldwide. The etiology of pulmonary extranodal MZL has yet to be elucidated (7), but chronic immune stimulation resulting from infection or autoimmune disorder may be associated with the pathogenesis of extranodal MZL. In addition, previous studies have identified the strong association between Helicobacter pylori infection and gastric extranodal MZL $(2,4)$.

It has been indicated that approximately half of patients are asymptomatic at presentation, with abnormal radiological findings being identified by chest radiography. For the initial staging and follow-up in patients with malignant lymphoma, ${ }^{18}$ Fluoro-2-deoxyglucose-positron emission tomography $\left({ }^{18}\right.$ FDG-PET) has been widely used (16). Although a previous study (17) indicated that there is a limited role for PET in extranodal MALT lymphoma patients due to the lack of FDG avidity, a later study by Elstrom et al (16) indicated that the issue is controversial, in particular, the accuracy of ${ }^{18} \mathrm{FDG}$ avidity in MZL. In the study by Elstrom et al, at least one site of involvement was detected by PET-FDG in only 67\% of MZL patients (16). Increased FDG uptake was detected in the majority of a small cohort of nodal MZL patients, but not in patients with extranodal disease, suggesting that the FDG-avidity depends on the tumor location or the MZL subtype (18).

In addition, symptomatic patients present with non-specific pulmonary symptoms, including cough, dyspnea, chest pain and hemoptysis. The B symptoms are uncommon and are observed in only a minor proportion of patients $(4,7,19,20)$.

In the current study, the patient with pulmonary extranodal MZL was asymptomatic and the pulmonary lesions were incidentally found during physical examinations at a local hospital. The major radiographic patterns of pulmonary extranodal MZL have been reported as nodules, consolidation, ground-glass opacity and centrilobular nodules with linear branching opacities, also termed a tree-in-bud sign $(4,7,15,20)$. In a previous study on the CT findings of MZL, single or multiple nodules or areas of consolidation were the main patterns, while none exhibited involvement of the main bronchus (15). In a later study, only two of the 61 enrolled patients were found to possess masses of various sizes in the main bronchus (19). Therefore, the characteristics of the present patient are observed extremely rarely in clinical settings as the patient presented with endobronchial edema and inflammatory symptoms, without lung parenchymal lesions being observed during bronchoscopy, which highly enhanced the difficulty of diagnosis and delayed the administration of the corresponding therapies.

Extranodal MZL can be clinically diagnosed by bronchoscopic, transbronchial or percutaneous needle biopsies, with a surgical lung biopsy being required in numerous cases $(4,15,19)$. An infiltrate of small to medium-sized lymphocytes with irregular nuclei and abundant cytoplasm is characteristic of extranodal MZL, with reactive follicles also usually observed. Lymphoepithelial lesions, in which tumor cells infiltrate the bronchial, bronchiolar and alveolar epithelium, are characteristic of MZL, but not pathognomonic $(2,4,7)$. Immunophenotyping studies aid in the confirmation of diagnosis, particularly when performed on small biopsy specimens, and aid in the differentiation of extranodal MZL from diffuse large B-cell, small lymphocytic, mantle cell and follicular lymphomas $(2,4,7,19)$. Pulmonary extranodal MZL is an indolent disease and exhibits a favorable prognosis, with a five-year survival rate of $\sim 90 \%(4,7,20)$, but extra-pulmonary lesions and lymph node involvement are poor prognostic factors (20).

In the present study, since the male patient was asymptomatic, which significantly increased the difficulty of the clinical diagnosis, the male patient was required to undergo a series of clinical examinations, comprising chest radiography, chest CT, bronchoscopic examinations, immunohistochemical staining and transbronchial lung biopsy. Combining these clinical results and subsequent analysis outcomes, an accurate diagnosis of pulmonary extranodal MALT-MZL was finally confirmed.

\section{References}

1. Harris NL, Jaffe ES, Stein H, et al: A revised European-American classification of lymphoid neoplasms: a proposal from the International Lymphoma Study Group. Blood 84: 1361-1392, 1994.

2. Armitage JO and Weisenburger DD: New approach to classifying non-Hodgkin's lymphomas: clinical features of the major histologic subtypes. Non-Hodgkin's Lymphoma Classification Project. J Clin Oncol 16: 2780-2795, 1998.

3. Zucca E, Conconi A, Pedrinis E, et al: Nongastric marginal zone B-cell lymphoma of mucosa-associated lymphoid tissue. Blood 101: 2489-2495, 2003.

4. Jaffe ES, Harris NL, Stein H and Vardiman JW (eds): World Health Organization Classification of Tumours. Pathology and Genetics of Tumours of Haematopoietic and Lymphoid Tissues. IARC Press, Lyon, pp171-181, 2001.

5. Ahmed S, Siddiqui AK and Rai KR: Low-grade B-cell bronchial associated lymphoid tissue (BALT) lymphoma. Cancer Invest 20: 1059-1068, 2002.

6. Koss MN: Malignant and benign lymphoid lesions of the lung. Ann Diagn Pathol 8: 167-187, 2004.

7. Travis WD, Brambilla E, Müller-Hermelink HK and Harris CC (eds): World Health Organization Classification of Tumours. Pathology and Genetics of Tumours of the Lung, Pleura, Thymus and Heart. 3rd edition. IARC Press, Lyon, pp95-98, 2004.

8. Cordier JF, Chailleux E, Lauque D, et al: Primary pulmonary lymphomas. A clinical study of 70 cases in nonimmunocompromised patients. Chest 103: 201-208, 1993.

9. Wislez M,Cadranel J, Antoine M, Milleron B, Bazot M, Mayaud C and Carette MF: Lymphoma of pulmonary mucosa-associated lymphoid tissue: CT scan findings and pathological correlations. Eur Respir J 14: 423-429, 1999.

10. Cadranel J, Wislez M and Antoine M: Primary pulmonary lymphoma. Eur Respir J 20: 750-762, 2002.

11. Zhang LB, Sun YE, Yu CH and Liu Y: Clinical diagnosis and surgical treatment of primary pulmonary lymphoma. Zhonghua Wai Ke Za Zhi 44: 97-99, 2006 (In Chinese).

12. Vanden Eynden F, Fadel E, de Perrot M, et al: Role of surgery in the treatment of primary pulmonary B-cell lymphoma. Ann Thorac Surg 83: 236-240, 2007.

13. Li G, Hansmann ML, Zwingers T and Lennert K: Primary lymphomas of the lung: morphological, immunohistochemical and clinical features. Histopathology 16: 519-531, 1990.

14. Isaacson P and Wright DH: Malignant lymphoma of mucosaassociated lymphoid tissue. A distinctive type of B-cell lymphoma. Cancer 52: 1410-1416, 1983.

15. Bae YA, Lee KS, Han J, et al: Marginal zone B-cell lymphoma of bronchus-associated lymphoid tissue: imaging findings in 21 patients. Chest 133: 433-440, 2008.

16. Elstrom R, Guan L, Baker G, et al: Utility of FDG-PET scanning in lymphoma by WHO classification. Blood 101: 3875-3876, 2003.

17. Perry C, Herishanu Y, Metzer U, et al: Diagnostic accuracy of PET/CT in patients with extranodal marginal zone MALT lymphoma. Eur J Haematol 79: 205-209, 2007.

18. Hoffmann M, Kletter K, Becherer A, et al: 18F-fluorodeoxyglucose positron emission tomography (18F-FDG-PET) for staging and follow-up of marginal zone B-cell lymphoma. Oncology 64: 336-340, 2003. 
19. Oh SY, Kim WS, Kim JS, et al: Pulmonary marginal zone B-cell lymphoma of MALT type-what is a prognostic factor and which is the optimal treatment, operation, or chemotherapy?: Consortium for Improving Survival of Lymphoma (CISL) study. Ann Hematol 89: 563-568, 2010.
20. Ahmed S, Kussick SJ, Siddiqui AK, et al: Bronchial-associated lymphoid tissue lymphoma: a clinical study of a rare disease. Eur J Cancer 40: 1320-1326, 2004. 